\title{
The properties of V838 Monocerotis in 2002 November
}

\author{
Ya. V. Pavlenko ${ }^{1,2}$, J. Th. van Loon $^{3}$, A. Evans ${ }^{3}$, M. T. Rushton ${ }^{3}$, B. M. Kaminsky², A. V. Filippenko ${ }^{4}$, R. J. Foley ${ }^{4}$, \\ W. $\mathrm{Li}^{4}$, B. Smalley ${ }^{3}$, and L. A. Yakovina ${ }^{2}$
}

${ }^{1}$ Centre for Astrophysics Research, University of Hertfordshire, College Lane, Hatfield, Hertfordshire AL10 9AB, UK e-mail: yp@mao.kiev.ua

2 Main Astronomical Observatory, Academy of Sciences of the Ukraine, Golosiiv Woods, Kyiv-127, 03680, Ukraine

3 Astrophysics Group, School of Physical \& Geographical Sciences, Keele University, Keele Staffordshire, ST5 5BG, UK

4 Department of Astronomy, 601 Campbell Hall, University of California, Berkeley, CA 94720-3411, USA

Received 26 April 2006 / Accepted 4 September 2006

\section{ABSTRACT}

\begin{abstract}
We present the results of modelling the $0.45-1 \mu \mathrm{m}$ spectral energy distribution of V838 Mon for 2002 November. Synthetic spectra were calculated using the NextGen model atmospheres of Hauschildt et al. (1999, ApJ, 512, 377), which incorporate line lists for $\mathrm{H}_{2} \mathrm{O}$, $\mathrm{TiO}, \mathrm{CrH}, \mathrm{FeH}, \mathrm{CO}$, and $\mathrm{MgH}$, as well as the VALD atomic line list. Fits to the observed spectra show that, in 2002 November, the effective temperature of V838 Mon was approximately $2000 \pm 100 \mathrm{~K}$. Our theoretical spectra show a comparatively weak dependence on $\log g$. Preliminary analysis of the hot star observed together with V838 Mon shows it to be a normal B3V dwarf.
\end{abstract}

Key words. stars: individual: V838 Mon- stars: atmospheres - stars: evolution

\section{Introduction}

V838 Mon (also known as EQJ0704.0-0350, GSC 04822-00039, IRAS 07015-0346, USNO-A2.0 0825-03833116, AAVSO 065903) has been the subject of intense interest since its discovery as an eruptive variable by Brown (2002) on January 6, 2002. Two further maxima on the light curve subsequently occurred, in 2002 February (Munari et al. 2002; Kimeswenger et al. 2002; Crause et al. 2003) and the optical flux in the $V$ band increased by $\sim 9$ mag. The luminosity peaked at $V \approx 7$ in 2002 February. Later, a gradual decline in the $V$ flux began which, by 2003 January, had faded by 8 mag.

Henden et al. (2002) reported the presence of a light echo, subsequently studied in detail by Bond et al. (2003) and Crause et al. (2005). The reflecting dust may be interstellar (Tylenda et al. 2005) or may have originated in the envelope of V838 Mon and ejected in the past (van Loon et al. 2004). Van Loon et al. (2004) reported the discovery of the multiple dust shells around V838 Mon. Alternatively, these shells arose through heavy mass loss during the evolution of a massive progenitor (Munari et al. 2005).

The distance to V838 Mon can be estimated from the evolution of the light echo (Henden et al. 2002). According to works based on Hubble Space Telescope data, its distance is $>6 \mathrm{kpc}$ (Bond et al. 2003), $8 \pm 2 \mathrm{kpc}$ (Tylenda 2004), or as high as $12 \mathrm{kpc}$ (Crause et al. 2005). However, according to recent work based on HST data, its distance is $5.9 \mathrm{kpc}$ (Sparks et al. 2006). If these estimates are correct, then at the time of maximum brightness V838 Mon was the most luminous star in the Galaxy.

The cause of the eruption of V838 Mon and the nature of its progenitor are unclear. The integrated colors of the progenitor looked that of an unreddened F-type star (Munari et al. 2002). Desidera \& Munari (2002) discovered spectroscopically a hot companion to the outbursting star, later confirmed by Wagner et al. (2002), and classified as a B3V star by Munari et al. (2005).
The spectral, photometric, and polarimetric evolution of V838 Mon has been described in several studies (Munari et al. 2002; Kimeswenger et al. 2002; Kolev et al. 2002; Wisniewski et al. 2003; Osiwała et al. 2003; Crause et al. 2003; Kipper et al. 2004; Rushton et al. 2005b). By mid-April 2002, bands of TiO had appeared in the spectrum and by May, the spectrum had evolved to a "very cold" M giant (Banerjee \& Ashok 2002). Its infrared spectrum in 2002 October was characterized as an "L-supergiant" by Evans et al. (2003).

The complexity and the rarity of V838 Mon optical spectrum resulted in only a few sporadic attempts to derive the star's atmospheric properties. Kipper et al. (2004) found that, for the iron group of elements, $[\mathrm{M} / \mathrm{H}]=-0.4$, while abundances of lithium and of some s-process elements are clearly enhanced. Later, Kaminsky \& Pavlenko (2005) provided fits to echelle data and obtained a similar result, $[\mathrm{Fe} / \mathrm{H}]=-0.3 \pm 0.2$. These results were obtained using a static LTE model and are therefore very dependent on the model atmosphere and spectrum synthesis assumptions.

In this paper we discuss fits to the spectral energy distribution of V838 Mon using data obtained in 2002 November. The observational data used in this paper are described in Sect. 2. Section 3 explains some of the background to our work and some details of the procedure and input parameters used. The results are discussed in Sect. 5.

\section{Observations}

The spectrum we consider was obtained on 2002 November 6 with the Kast spectograph on the Cassegrain focus of the Shane 3-m telescope at Lick Observatory. The resolution of the spectrum is $\lambda / \Delta \lambda=3000$. The observing and data reduction processes are the same as those in Rushton et al. (2005b) and are not repeated here. 


\section{Computation of theoretical spectra}

Theoretical spectral energy distributions (SEDs) were computed for model atmospheres of giants from the NextGen grid of Hauschildt et al. (1999), with solar metallicity (Anders \& Grevesse 1989).

In our computations we used a number of model atmospheres, with $T_{\text {eff }}=2000-2200 \mathrm{~K}$ and $\log g=0,0.5$. Computations of the synthetic spectra were carried out using the program WITA6 (Pavlenko 2000), assuming LTE and hydrostatic equilibrium for a one-dimensional model atmosphere without sources and sinks of energy.

The equations of ionization-dissociation equilibrium were solved for media consisting of atoms, ions, and molecules. We took into account $\sim 100$ components (Pavlenko 2000). The constants for the equations of chemical balance were taken from Tsuji (1973). Molecular line data were taken from a variety of sources:

(i) the TiO line lists of Plez (1998);

(ii) CN lines from CDROM 18 (Kurucz 1993);

(iii) $\mathrm{CrH}$ and $\mathrm{FeH}$ lines from Burrows et al. (2002) and Dulick et al. (2003), respectively;

(iv) lines of $\mathrm{H}_{2}^{16} \mathrm{O}$ (Barber et al. 2006);

(v) absorption by VO, and by a few molecules of (in the case of V838 Mon) lesser importance, was computed in the JOLA approximation (see Pavlenko 2000); and

(vi) atomic line list from VALD (Kupka et al. 1999).

The profiles of molecular and atomic lines are determined using the Voigt function $H(a, v)$. Their natural $\left(C_{2}\right)$ and van der Waals broadening $\left(C_{6}\right)$ parameters are taken from Kupka et al. (1999) or, in their absence, computed following Unsold (1955). Owing to the low temperatures in M-star atmospheres, and the consequent low electron densities, Stark broadening may be neglected; on the whole the effects of pressure broadening dominate. Computations for synthetic spectra were carried out with a step of $0.003 \mu \mathrm{m}$, for microturbulent velocity $v_{\mathrm{t}}=5 \mathrm{~km} \mathrm{~s}^{-1}$.

There are several reasons to suspect that dust might be present in the atmosphere of V838 Mon, see discussion in Banerjee \& Ashok (2002); Evans et al. (2003); Rushton et al. (2005a). However, examination of the results of preliminary runs of our models without dust show that the density of condensibles (such as X, Y, Z) at the appropriate distance is low, so that precipitation in the photosphere is extremely unlikely. Hence, we do not include dust in our models.

In addition, we do not take account of the effects on the model atmosphere of the presence of a B3 star (Munari et al. 2005). Over the wavelength range we are modelling (6000$10000 \AA$ ), the SED is dominated by the cool star and the contribution of the B3 star is negligible. There may be irradiation effects to be taken into account if the hot component is part of a V838 Mon binary system, but we do not pursue this here. We will, however, include the effects of the B3 star to the overall SED after fitting the red end of the spectrum (see Sect. 4.4 below).

The relative importance of the different opacities contributing to our synthetic spectra is shown in Fig. 1.

\section{Results}

\subsection{Dependence of fits on input parameters}

Changes in our input parameters, i.e. $T_{\text {eff }}, \log g,[\mathrm{Fe} / \mathrm{H}], v_{\mathrm{t}}$, affect the theoretical spectral distributions (see Fig. 2) in different ways, as follows: (i) The response of the spectrum to changes in $\log g$ is relatively weak due to the absence of strong atomic lines with extended wings. Due to low densities in the atmosphere of V838 Mon pressure broadening cannot be significant. Saturated $\mathrm{TiO}$ and VO band heads show rather low sensitivity on $\log g$;

(ii) Due to the same reason, changes in the microturbulent velocity mainly affect the heads of strong molecular bands;

(iii) On the other hand, changes in metallicity reduce the molecular absorption over the entire spectral region;

(iv) Due to the low temperatures in the atmosphere of V838 Mon changes in the effective temperature affect the slope of the computed spectra in the region 0.6-1 $\mu \mathrm{m}$. This effect can be used to determine the effective temperature of V838 Mon (see Sect. 4.3).

Comparison of the computed spectrum with that of V838 Mon in 2002 November suggests that the observed fluxes at $\lambda>0.9$ and $\lambda<0.8 \mu \mathrm{m}$ are better fitted with a slightly metal-deficient atmosphere $([\mathrm{Fe} / \mathrm{H}=-0.5)$. This is in agreement with the analysis by Kipper et al. (2004) and Kaminsky \& Pavlenko (2005).

\subsection{Comparison with the spectrum of Kelu1 (L2)}

We compare (see Fig. 1) the observed spectrum of V838 Mon with that of the brown dwarf Kelu1 (Leggett et al. 2002). The L2 brown dwarf Kelu1 has approximately the same effective temperature as V838 Mon but, by definition, its $\log g$ is very much higher. There are several significant points to note:

(i) Heads of VO bands are clearly seen on at $0.74,0.79,0.87$ and $0.96 \mu \mathrm{m}$;

(ii) The molecular bands of $\mathrm{TiO}$ and $\mathrm{VO}$ in the spectrum of V838 Mon are very intense. Due to the low densities these species are practically undepleted in the atmosphere of V838 Mon despite the low temperatures. On the other hand, $\mathrm{Ti}$ and $\mathrm{V}$ containing species are completely depleted in the atmospheres of L-dwarfs;

(iii) The pressure broadened $\mathrm{K} \mathrm{I}$ and $\mathrm{Na} \mathrm{I}$ lines are prominent in the optical spectra of L dwarfs (Pavlenko 2001). Instead of L-dwarfs spectra the alkali lines are absent or very weak in the spectrum of V838 Mon;

(iv) The electronic bands of $\mathrm{CrH}$ and $\mathrm{FeH}$ are much weaker in the spectrum of V838 Mon than they are in Kelu1.

\subsection{The effective temperature of V838 Mon in 2002}

A comparison of the observed spectrum of V838 Mon with theoretical spectra computed using NextGen model atmospheres is presented in Fig. 3, in which we use a logarithmic scale for the purpose of illustration as the observed fluxes cover several orders of magnitude. The instrumental broadening was modelled by Gaussian profiles set to the resolution of the observed spectra $(F H W M=2 \AA)$. The spectrum was dereddened by $E(B-V)=0.87 \mathrm{mag}$ (Munari et al. 2005).

The following are worth noting:

(i) We can fit the overall slope of the spectrum in the nearIR/optical region, together with most of the spectral features. The spectrum is dominated by $\mathrm{TiO}$ bands, although some bands of VO are also prominent;

(ii) This provides clear evidence that, on 2002 November 6 , $\mathrm{C} / \mathrm{O}<1$ in V838 Mon; 

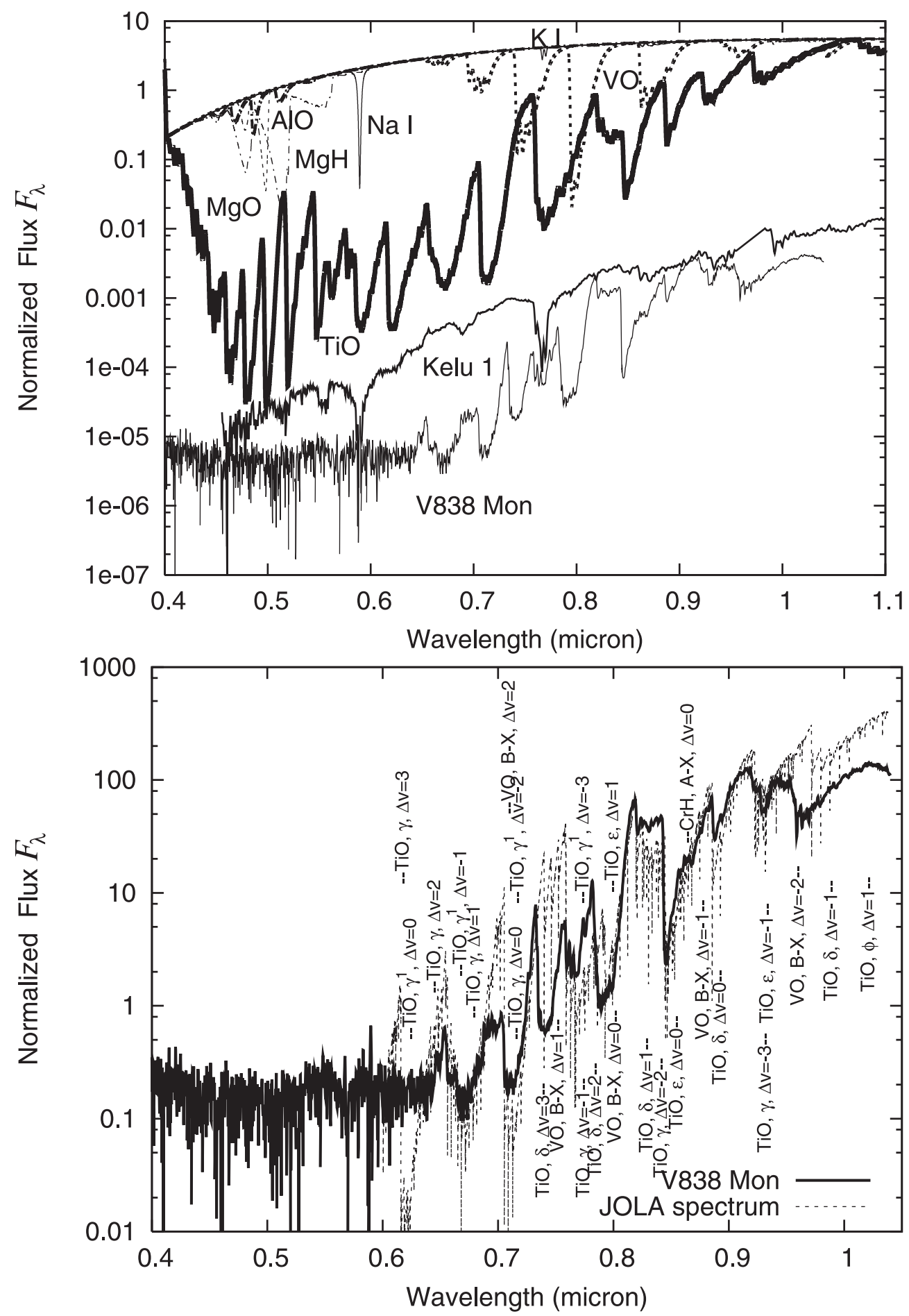

Fig. 1. Top: contribution of different molecules to the formation of the spectrum of V838 Mon. The two lower spectra represent observed fluxes of L-dwarf Kelu1 (Leggett et al. 2002) and V838 Mon. Bottom: identification of the main molecular features in the spectrum of V838 Mon. Data for the figure plotting and color version of the plots are available on $\mathrm{ftp}: / / \mathrm{ftp} . \mathrm{mao} . \mathrm{kiev} . \mathrm{ua} / \% 2 \mathrm{f} / \mathrm{pub} / \mathrm{users} / \mathrm{yp} / \mathrm{Fig} .1$.

(iii) The dependence on $\log g$ is rather weak, as expected (see Sect. 4.1). Strong atomic lines are practically absent in the spectrum;

(iv) The dependence on $T_{\text {eff }}$ is much stronger. From comparison of the computed and observed SEDs, we conclude that the effective temperature of V838 Mon was $T_{\text {eff }}=$ $2000 \pm 100 \mathrm{~K}$ in 2002 November.

Our value of $T_{\text {eff }}$ for 2002 November 6 compares favourably with that determined by Tylenda (2005) for 2002 day 301
(October 28, the nearest in Tylenda's compilation); he obtained $T_{\text {eff }}=2180 \mathrm{~K}$ (formally this corresponds to a spectral class M8.5).

\subsection{The hot component in the V838 Mon spectrum}

Our observed spectrum of V838 Mon shows a rise in the flux toward the blue end of the spectrum, at $\lambda \lesssim 6500 \AA$, which can be attributed to the presence of the "hot" star; this may be a background or foreground object, or even a member of a V838 Mon 

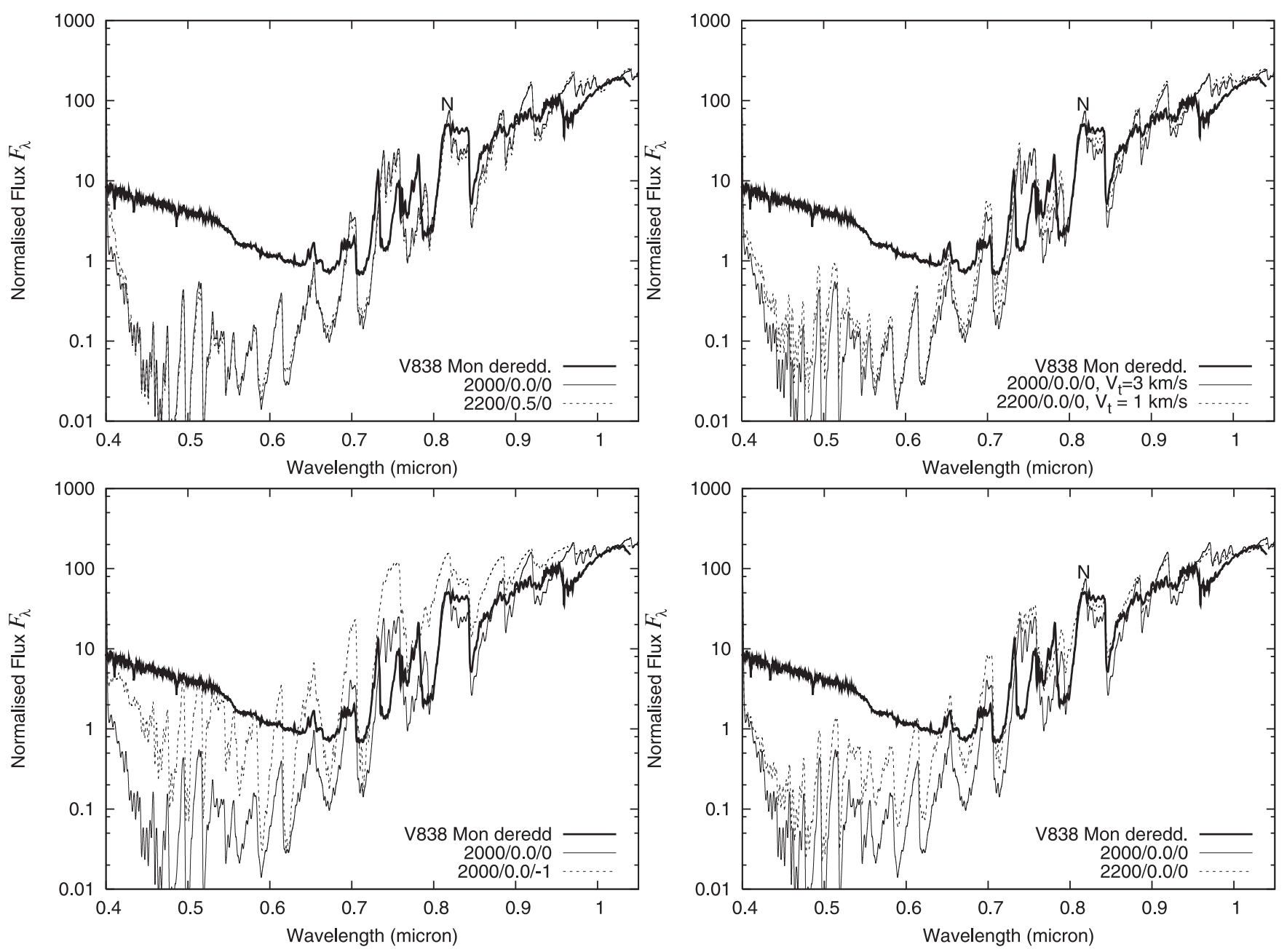

Fig. 2. Dependence of computed spectra on the gravity (top left), microturbulent velocity (top right), metallicity (bottom left), and effective temperature (bottom right). The dereddened observed spectrum of V838 Mon is shown by the boldfaced line. Model atmospheres are labeled on the figure as $T_{\text {eff }} / \log /[\mathrm{M} / \mathrm{H}]$, i.e. $2000 / 0.0 /-1$ means $T_{\text {eff }}=2000, \log g=0.0,[\mathrm{M} / \mathrm{H}]=-1$.

binary system (see discussion in Munari et al. 2005). It is worth noting that all the Balmer hydrogen lines, from $\mathrm{H} \beta$ to the Balmer jump, are clearly seen in the observed spectrum.

We first model the contribution of the hot component by a simple Rayleigh-Jeans law $F_{\lambda}=$ const. $/ \lambda^{4}$ and normalise both fluxes to the same value at $0.67 \mu \mathrm{m}$. This comparatively simple procedure allows us to fit to the observed spectrum over a wide spectral range, from the blue to the near-infrared.

We next computed a set of theoretical spectra using the standard Kurucz model and WITA6, for different values of $T_{\text {eff }}$ and $\log g$. A comparison of the observed spectrum of the hot companion with theoretical spectra is presented in Fig. 4. Unfortunately, our low-resolution spectrum, with its low signalto-noise ratio in the blue, cannot provide a reliable estimate of the atmospheric parameters (effective temperature, gravity and/or metallicity) of the hot component. From the fit to the observed spectrum we estimate $T_{\text {eff }}=18000-23000 \mathrm{~K}$ and $\log g=4.0$.

From evolutionary calculation (Schaller et al. 1992) we find the mass of the B star; we then computed the possible range of spectral parallaxes for our hot component. We obtain $M=6 M_{\odot}$, $\mathrm{d}=7.2 \mathrm{kpc}$ for $T_{\mathrm{eff}}=18000$ and $M=9 M_{\odot}, d=14.4 \mathrm{kpc}$ for $T_{\text {eff }}=23000 \mathrm{~K}$, respectively, i.e. only in the case of $T_{\text {eff }}<$ $21000 \mathrm{~K}$ the hot component can be a member of the V838 Mon binary system.

\section{Discussion and conclusions}

It seems that the pseudophotosphere of V838 Mon was evolving sufficiently slowly that its optical spectrum could be approximated by a sequence of classical, i.e. static model atmospheres (Kaminsky \& Pavlenko 2005); we find that the standard computational procedures for SEDs, applied to V838 Mon, give results that are reliable enough to determine the effective temperature. It is worth noting that the presence of $\mathrm{P}$ Cyg profiles indicates expansion velocities up to $200 \mathrm{~km} \mathrm{~s}^{-1}$. However the emission components of the P Cyg profiles form at the outer boundary of the envelope, well above the region where the observed continuum and absorption lines form.

There are distinct differences between the SED of V838 Mon and of late-type dwarfs with similar spectral type, which obviously arise from their differing physical nature and the input physical data. In the case of V838 Mon we have modelled its SED at an advanced stage of its evolution and find that its optical spectrum is formed by absorption of the saturated bands of $\mathrm{VO}$ and $\mathrm{TiO}$.

We note that our models do not satisfactorily reproduce the absorption band located around $0.75 \mu \mathrm{m}$; this absorption cannot be identified as $\mathrm{TiO}$ or VO bands (see Fig. 1). Hypothetically, the strong band of $\mathrm{CH}_{4}$ could be formed in a low-temperature 


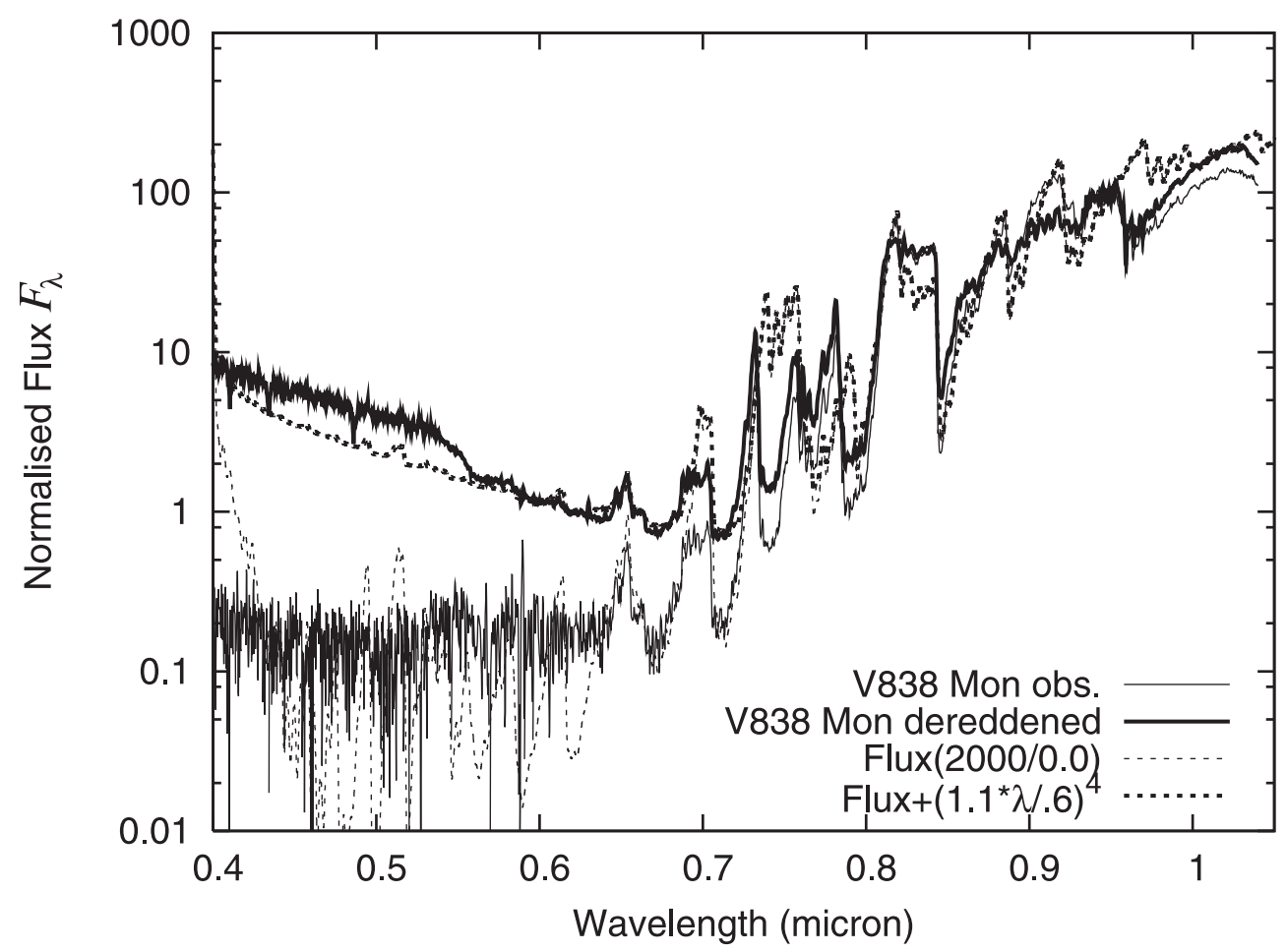

Fig. 3. Fit of theoretical fluxes to the observed spectrum of V838 Mon. Observed and dereddened spectra are shown by solid thin and thick lines, respectively. The computed V838 Mon spectrum is shown by thin dashed line. The combined spectrum (computed V838 Mon + Rayleigh-Jeans contribution from the B star) is shown by the thick dahsed line. The color version of the plot is available on ftp://ftp.mao.kiev.ua/\%2f/pub/users/yp/Fig.3.
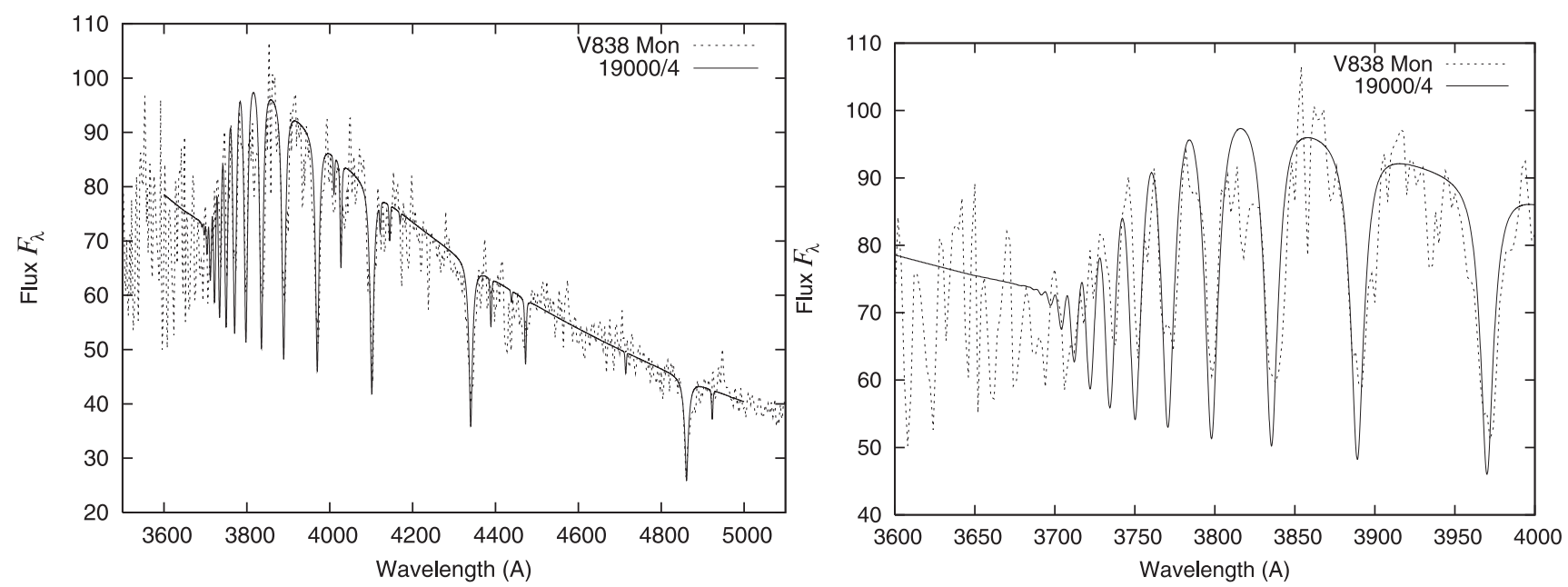

Fig. 4. Left: fit of the theoretical spectrum with $T_{\text {eff }}=19000 \mathrm{~K}$ and $\log g=4.0$ to the hot companion of V838 Mon. Right: the Balmer jump region of the hot companion spectrum. To simplify the picture we exlude the metal line absorption from the accounted opacity sources.

regime at these wavelengths. This band, as well as the bands at 0.619 and $0.890 \mu \mathrm{m}$, are known to be present in the spectra of Jupiter and other gas giants. Possibly the band may be formed in the outermost layers of the extended envelope of V838 Mon. We plan to provide a more detailed study of the molecular features in a forthcoming paper.

Nonetheless, we have obtained a qualitatively good fit of the spectrum of V838 Mon over a wide spectral range $(0.4-1 \mu \mathrm{m})$ with a model atmosphere having $T_{\text {eff }}=2000 \pm 100 \mathrm{~K}$ and $\mathrm{C} / \mathrm{O}<$ 1 , and a hot component consistent with $T_{\text {eff }}=18000-23000 \mathrm{~K}$ and $\log g=4.0 \pm 0.5$; this agrees well with previous studies
(Tylenda 2005). The cool component is slightly better reproduced with a moderately sub-solar metallicity model.

Moreover the computation of the SED was carried out within the framework of the classical approach: there is not at this stage any need to include additional physical processes to find the best fit to the observed SEDs.

Unfortunately, from our study we cannot determine other basic parameters, i.e. $v_{\mathrm{t}}, \log g$ and $[\mathrm{Fe} / \mathrm{H}]$. The main features in our low resolution spectra are formed by the heads of saturated molecular bands. These spectral features show rather low sensitivity on small variations of $v_{\mathrm{t}}, \log g$ and $[\mathrm{Fe} / \mathrm{H}]$. They can be determined from the fits to high resolution spectra. 
Our study enables us to draw the following conclusions:

(i) We can use fits to SEDs of V838 Mon to determine its basic parameters; in particular the effective temperature $T_{\text {eff }}=$ $2000 \pm 100 \mathrm{~K}$;

(ii) We clearly see that, in 2002 November $6, \mathrm{C} / \mathrm{O}<1$ in the atmosphere of V838 Mon;

(iii) The contribution of the hot star to the spectrum of V838 Mon is clearly seen in 2002 November; this hot component is consistent with $T_{\text {eff }}=19000 \mathrm{~K}$ and $\log g=4.0$.

Acknowledgements. Y.P.'s work was partially supported by the Royal Society and the Leverhulme Trust. The computations were in part performed using the resources of HiPerSPACE computing facility at UCL which is part funded by the UK Partical Physics and Astronomy Research Council (PPARC). A.V.F. acknowledges support from USA NSF Grant AST-0307894. We thank anonymous referee for the helpful comments.

\section{References}

Anders, E., \& Grevesse, N. 1989, GeGoAA, 53, 197

Banerjee, D. P. K., \& Ashok, N. M. 2002, A\&A, 395, 161

Barber R. J., Tennyson J., Harris G. J., \& Tolchenov R. 2006, MNRAS, 368, 107

Bond, H. E., Henden, A., Levay, Z. G., et al. 2003, Nature, 422, 405

Brown, N. J. 2002, IAUC 7785

Burrows, A., Ram, S. R., \& Bernath, P. 2002, ApJ, 577, 986

Crause, L. A., Lawson, W. A., Kilkenny, D., et al. 2003, MNRAS, 341, 785

Crause, L. A., Lawson, W. A., Menzies, J. W., \& Marang, F. 2005, MNRAS, 358, 1352

Desidera, S., \& Munari, U. 2002, IAUC7982

Dulick, M., Bauschlincher, C. W., \& Burrows, A. 2003, ApJ, 594, 651

Evans, A., Geballe, T. R., Rushton, M., T., et al. 2003, MNRAS, 343, 1054

Hauschildt, P. H., Allard, F., \& Baron, E. 1999, ApJ, 512, 377

Henden, A., Munari, U., \& Schwartz, M.B. 2002, IAUC7859
Kaminsky, B. M., \& Pavlenko, Y. P. 2005, MNRAS, 357, 38

Kimeswenger, S., Lederle, C., Schmeja, S., \& Armsdorfer, B. 2002, MNRAS, 336, L43

Kipper, T., Klochkova, V.G., Annuk, K., et al. 2004, A\&A, 416, 1107

Kolev, D., Mikolajevski, M., Tomov, T., et al. 2002, Collected Papers Physics, Shumen Univers. Press, 147

Kupka, F., Piskunov, N., Ryabchikova, T. A., Stempels, H. C., \& Weiss, W. W. 1999, A\&AS, 138, 119

Kurucz, R. L. 1993, CDROMs 1-22, Harvard-Smisthonian Astonomical Observatory

Leggett, S. K., Golimowski, D.A., \& Fan, X. 2002, ApJ, 564, 452

McLean, I. S., McGovern, M. R., Burgasser, A. J., et al. 2003, ApJ, 596, 561

Munari, U., Henden, A., Kiyota, S., et al. 2002, A\&A, 389, L51

Munari, U., Henden, A., Valenari, A., et al. 2005, A\&A, 434, 1107

Osiwała, J. P., Mikołajewski, M., Tomov, T., et al. 2003, in Symbiotic Stars Probing Stellar Evolution, ed. R. L. M. Corradi, R. Mikołajewska, T. J. Mahoney, San Francisco, ASP Conf. Proc., 303, 240

Pavlenko, Ya. 2000, Astron. Rep., 44, 219

Pavlenko, Ya. 2001, Astron. Rep., 45, 144

Pirzkal, N., \& Freudling, W. 1998, Nicmos and the VLT

Plez, B. 1998, A\&A, 337, 495

Rushton, M. T., Geballe, T. R., Evans, A., et al. 2005a, MNRAS, 359, 624

Rushton, M. T., Geballe, T. R., Filippenko, A. V., et al. 2005b, MNRAS, 360, 1281

Sparks, B. 2006, in The nature of v838 Mon and its light echo Conf. Proc., La Palma, May 15-19, ed. U.Munary and R. Corrado, in press

Schaller, G., Schaerer, D., Meynet, G., \& Maeder, A., 1992, A\&AS, 96, 269

Tsuji, T. 1973, A\&A, 23, 411

Tsuji, T., Ohnaka, K., \& Aoki, W. 1997, ed. H. Okuda, T. Matsumoto, T. Rollig, ASP Conf. Ser. 124, 91

Tylenda, R., 2004, A\&A, 414, 223

Tylenda, R., 2005, A\&A, 436, 1009

Tylenda, R., Soker, N., \& Szczerba, R. 2005, A\&A, 441, 1099

Unsold, A. 1955, Physik der Sternatmospheren, 2nd ed. (Berlin: Springer)

van Loon, J. Th., Evans, A., Rushton, M.T., \& Smalley, B. 2004, A\&A, 427, 193

Wisniewski, J. P., Morrison, N. D., Bjorkman, K. S., et al. 2003, ApJ, 588, 486 